\title{
Потенциометрическое определение треонина в кислых растворах с помощью мембран МФ-4СК с сульфосодержащими допантами
}

\author{
Титова T.С., Рыжих Е.А., Паршина А.В., Бобрешова О.В. \\ ФГБОУ ВО «Воронежский государственный университет», Воронеж
}

Поступила в редакцию 13.07.2017 г.

\begin{abstract}
Разработаны перекрестно чувствительные ПД-сенсоры (аналитический сигнал - потенциал Доннана) на основе мембран МФ-4СК с оксидами, поверхностно модифицированными сульфосодержащими фрагментами, в кислых растворах треонина. Выявлена корреляция между чувствительностью ПД-сенсоров к ионам треонина и гидроксония и объемной доли вводимых в мембрану допантов. Наибольшая чувствительность ПД-сенсоров к ионам аналита и наименьшая к мешающим ионам достигается при использовании образцов, содержащих 3-5 мас. \% диоксида кремния, модифицированого 3-пропилсульфокислотными группами. Использование данных мембран обеспечило снижение относительной погрешности определения катионов и цвиттерионов треонина в диапазоне концентраций от $1.0 \cdot 10^{-4}$ до $1.0 \cdot 10^{-1} \mathrm{M}(\mathrm{pH} 1.73-4.25)$ в $2.5-4$ раза по сравнению с исходным образцом.

Ключевые слова: потенциометрические сенсоры, перекрестная чувствительность, потенциал Доннана, перфторированные сульфокатионообменные мембраны, гибридные материалы, функционализация поверхности допантов, треонин.
\end{abstract}

\section{Potentiometric determination of threonine in acid solutions by used MF-4SC membranes with sulfo-containing dopants}

\author{
Titova T.S., Ryzhih E.I., Parshina A.V., Bobreshova O.V. \\ Voronezh State University, Voronezh
}

\begin{abstract}
Amino acids are components of pharmaceuticals, products for children and sports nutrition, and also they use as feed additives. The electrochemical methods, in particular dialysis and electrodialysis, are being developed for the separation and purification of amino acids. The necessity to control of processes efficiency and the quality of products in the production of amino acids necessitates the development of methods for their rapid determination, taking into account the variable medium $\mathrm{pH}$. The aim of this work was to reduce the influence of hydroxonium ions on the determination of threonine cations and zwitterions in aqueous solutions at $\mathrm{pH}<7$. For this, cross-sensitive DP-sensors (analytical signal is Donnan potential) based on MF-4SC membranes with oxides surface-modified with sulfo-groups were used.

The characteristics of DP-sensors were determined in the equimolar solutions of threonine (Thr) and hydrochloric acid with component concentrations from $1.0 \cdot 10^{-4}$ to $1.0 \cdot 10^{-1} \mathrm{M}$. In the $\mathrm{pH}$ range of the investigated solutions (1.73-4.25), threonine is present in the form of singly charged cations $\left(\mathrm{Thr}^{+}\right)$and zwitterions $\left(\mathrm{Thr}^{ \pm}\right)$. The perfluorosulfonic membranes (MF-4SC) and the hybrid materials based on them with nanoparticles of hydrated zirconia and silica, surface modified by the $-\left(\mathrm{CH}_{2}\right)_{3}-\mathrm{SO}_{3} \mathrm{H}$ and $-\mathrm{SO}_{3} \mathrm{H}$ sulfo containing fragments, were used as materials for DP-sensors. The samples of membranes were provided by the Laboratory of Ionics of Functional Materials of the Kurnakov Institute of General and Inorganic Chemistry RAS (the head of laboratory is Yaroslavtsev A.B., Dr.Sci. (Chemistry), prof., corresponding member of the RAS; a synthesis of materials is carried out by Safronova E.Yu., senior scientific researcher, Ph.D. (Chemistry)).
\end{abstract}


The influence of the concentration and proton-donor properties of dopants introduced into the membranes on the characteristics of cross-sensitive DP-sensors in threonine solutions at $\mathrm{pH}<7$ are investigated.It is shown, that the sensitivity of DP-sensors increases to cations and zwitterions of threonine and decreases to hydronium ions with an increase in the volume fraction of dopants in membranes due to a simultaneous decrease in the volume of the intraporous space and an increase in the number of binding sites for analyte ions. Under such conditions, a transition of the bulk $\mathrm{Thr}^{+}$and $\mathrm{Thr}^{ \pm}$ions is apparently possible, and their interaction with the sulfo-groups on the membrane pore walls and on the oxide surface excludes the part of protons from the ion exchange. The highest sensitivity of DP-sensors to analyte ions and the smallest sensitivity to interfering ion was obtained by the using samples containing 3-5 wt.\% of silica modified by $-\left(\mathrm{CH}_{2}\right)_{3}-\mathrm{SO}_{3} \mathrm{H}$ groups. Using these membranes provided a reduction of the relative error determination of threonine cations and zwitterions in 2.5-4 times compared with the initial sample in the concentration range of analyte from $1.0 \cdot 10^{-4}$ to $1.0 \cdot 10^{-1} \mathrm{M}$ (pH 1.73-4.25).

Keywords: potentiometric sensors, cross sensitivity, Donnan potential, perfluorosulfonic membranes, hybrid materials, functionalization of the dopant surface, threonine.

\section{Введение}

Аминокислоты являются компонентами фармацевтических препаратов, продуктов для детского и спортивного питания, а также применяются в качестве кормовых добавок. Для разделения и очистки аминокислот разрабатываются электрохимические методы, в частности диализ $[1,2]$ и электродиализ $[3,4]$. Необходимость контроля эффективности процессов и качества продуктов при получении аминокислот обусловливает необходимость разработки методов их экспресс-определения с учетом непостоянного рН среды. Не смотря на возрастающее количестворабот, посвященных химическим и биологическим сенсорам для определения аминокислот [5-9], проблеме влияния $\mathrm{pH}$ среды на аналитический сигнал и учета зависимости концентраций ионных форм аналита от рН раствора не уделяется должного внимания [10].

В работе [11] показана возможность нивелирования мешающего влияния ионов гидроксония на отклик ПД-сенсора (аналитический сигнал - потенциал Доннана) в кислых растворах аминокислоты с гидрофильным радикалом (на примере гистидина) путем введения в матрицу перфторированных сульфокатионообменных мембран МФ-4СК допантов с протонодонорными свойствами. Такая модификация позволяет снизить влагосодержание, увеличить ионную проводимость и, при определенном составе образцов, снизить диффузионную проницаемость по сравнению с исходной мембраной $[12,13]$. Оптимизация составов мембран, обеспечивающая одновременно возможность перехода в мембрану объемных органических катионов и увеличение селективности переноса катионов, приводит к увеличению концентрации ионов аналита в мембране, что препятствует взаимодействию протонов с функциональными группами мембраны и допанта [11]. Для определения катионов и цвиттерионов треонина, имеющего гидрофильный радикал, представляет интерес исследование влияния концентрации и протонодонорных свойств вводимых в мембраны допантов на перекрестную чувствительность и стабильность отклика ПД-сенсоров в соответствующих кислых растворах.

Целью работы являлась разработка перекрестно чувствительных ПД-сенсоров для определения катионов и цвиттерионов треонина в водных растворах при $\mathrm{pH}<7$ путем использования мембран МФ-4СК с оксидами, поверхностно модифицированными сульфогруппами.

\section{Эксперимент}

Реагенты и материалы. Использовали перфторированные сульфокатионообменные мембраны МФ-4СК и гибридные материалы на их основе, предоставленные 
Лабораторией ионики функциональных ИОНХ РАН (заведующий лабораторией д.х.н., проф., член.-корр. РАН Ярославцев А.Б., синтез материалов выполнены к.х.н, старшим научным сотрудником ИОНХ РАН Сафроновой Е.Ю.); аминокислоту треонин («Sigma-Aldrich», $\geq 98 \%$ ); хлорид калия (х.ч., «Химмед»); соляную кислоту (х.ч., «Химмед»); бидистиллированную воду (сопротивление 0.45 МОм).

Объектами исследования выбраны эквимолярные растворы треонина (Thr) и соляной кислоты с концентрациями компонентов от $1.0 \cdot 10^{-4}$ до $1.0 \cdot 10^{-1} \mathrm{M}$. В интервале $\mathrm{pH}$ исследуемых растворов (1.73-4.25) треонин присутствует в форме однозарядных катионов $\left(\mathrm{Thr}^{+}\right)$и цвиттерионов $\left(\mathrm{Thr}^{ \pm}\right)$.<smiles>CC(O)C([NH3+])C(=O)[O-]</smiles>

$\mathrm{Thr}^{ \pm}$<smiles>CC(O)C(N)C(=O)O</smiles>

$\mathrm{Thr}^{+}$

Гибридные материалы на основе мембран МФ-4СК получены отливкой из раствора полимера в присутствии заданного количества готовых частиц допантов. В качестве допантов выбраны гидратированные диоксиды кремния и циркония, поверхностно модифицированные сульфосодержащими фрагментами: $\mathrm{SiO}_{2}-\left(\mathrm{CH}_{2}\right)_{3}$ $\mathrm{SO}_{3} \mathrm{H}$ (обменная емкость $\sim 0.74$ ммоль/г) и $\mathrm{ZrO}_{2}-\mathrm{SO}_{3} \mathrm{H}$ (обменная емкость $\sim 1.1$ ммоль/г). Образцы кондиционировали для приведения к стандартным условиям, затем переводили в $\mathrm{K}^{+}$-форму, выдерживая в течение 72 часов в $2 \mathrm{M}$ растворе $\mathrm{KCl}$ с последующей промывкой в бидистиллированнойводе. Состав исследуемых образцов представлен в табл. 1. Массовую долю допантов определяли термогравиметрически $[11,12]$. Объемную долю рассчитывали исходя из массы и плотности вводимого в мембрану допанта. Методики получения и свойства данных мембран подробно описаны в работах $[11,12]$.

Таблица 1. Состав исследуемых мембран МФ-4СК $[11,12]$

\begin{tabular}{|c|c|c|}
\hline Допант & Массовая доля допанта, \% & Объемная доля допанта, \% \\
\hline- & - & 1.2 \\
\hline $\mathrm{ZrO}_{2}-\mathrm{SO}_{3} \mathrm{H}$ & 3 & 2.6 \\
\hline \multirow{2}{*}{$\mathrm{SiO}_{2}-\left(\mathrm{CH}_{2}\right)_{3}-\mathrm{SO}_{3} \mathrm{H}$} & 3 & 4.4 \\
\cline { 2 - 3 } & 5 & \\
\hline
\end{tabular}

В ПД-сенсорах использовали материалы, содержащие допант в одной части образца, конец которой в процессе эксперимента контактирует с раствором аналита. Отсутствие допанта в части, контактирующей с раствором сравнения, необходимо для обеспечения близости составов раствора внутри мембраны и раствора сравнения для нивелирования потенциала Доннана на границе раствор сравнения / мембрана.

Методы исследования. Электрохимическая ячейка для оценки электродноактивных свойств мембран разного состава включала четыре перекрестно чувствительных ПД-сенсора на их основе, стеклянный электрод (ЭС-10601/7) для контроля pH исследуемого раствора, электрод сравнения (ЭСр-10103) и многоканальный потенциометр. В электрохимической ячейке для определения треонина число перекрестно чувствительных ПД-сенсоров снижалось до одного. Электрохимическая цепь для оценки отклика ПД-сенсора на основе мембраны с градиентным распределением допанта по длине образца имеет вид:

$\mathrm{Ag}|\mathrm{AgCl}, 1 \mathrm{M} \mathrm{KCl}|$ мембрана / мембрана+допант $\mid$ исследуемый раствор $\mid$ нас. $\mathrm{KCl}$,

$$
\mathrm{AgCl} \mid \mathrm{Ag} \text {. }
$$

Для характеризации ПД-сенсоров определяли время, дрейф и дисперсию $\left(\mathrm{s}^{2}, \mathrm{MB}^{2}\right)$ отклика, чувствительность сенсоров, пределы обнаружения аналита 
$\left(\mathrm{c}_{\min }, \mathrm{M}\right)$, относительную погрешность $\left(\delta=\left(c_{\text {эксп }}-c_{\mathrm{BB}}\right) / c_{\mathrm{BB}}, \%\right.$, где $c_{\text {вв }}$ и $c_{\text {эксп }}-$ заданное и среднее найденное значения концентраций аналита) и относительное стандартное отклонение $\left(s_{\mathrm{r}}=s / c_{\text {эксп }} \%\right)$ определения треонина в исследуемых растворах. Значения времени и дрейфа отклика сенсоров определяли хронопотенциометрически. Для оценки перекрестной чувствительности ПД-сенсоров в растворах $\mathrm{Thr}+\mathrm{HCl}$ выбраны градуировочные уравнения следующего вида:

$$
\Delta \varphi_{D}=b_{0}+b_{1} \cdot p T h r+b_{2} \cdot p H
$$

где $\Delta \varphi_{D}$ - величина отклика ПД-сенсора, мB; $p T h r$ - отрицательный десятичный логарифм суммарной молярной концентрации катионов $T h r^{+}$ицвиттерионов $T h r^{ \pm} ; b_{0}-$ свободный член градуировочного уравнения, мВ; $b_{i}-$ коэффициенты чувствительности ПД-сенсора к соответствующим ионам, $\mathrm{MB} / \mathrm{pC}$. Для выявления возможных систематических ошибок и доказательства правильности выбора градуировочного уравнения оценивали его адекватность по F-критерию Фишера. Значимость коэффициентов уравнения оценивали по t-критерию Стьюдента. Пределы обнаружения аналита оценивали по правилу «три сигма», как минимальную концентрацию аналита, при которой величина отклика сенсора в растворе отличается от величины отклика в бидистиллированной воде $\left(\left(\Delta \varphi_{\mathrm{D}}\right)_{\text {фон }}, \mathrm{MB}\right)$ более, чем на утроенное стандартное отклонение отклика в бидистиллированной воде $\left(\mathrm{s}_{\text {фон }}, \mathrm{mB}\right)$.

\section{Обсуждение результатов}

Для определения ионов треонина в водных растворах при $\mathrm{pH}<7$ необходимы ПД-сенсоры с высокой чувствительностью к ним, низкой чувствительностью к мешающим ионам $\mathrm{H}_{3} \mathrm{O}^{+}$и высокой стабильностью отклика в исследуемых растворах.

Чувствительность ПД-сенсоров к объемным катионам и цвиттерионам определяется рядом факторов: возможностью их перехода в мембрану при установлении квазиравновесия на границе мембрана/раствор аналита, их концентрацией в мембране и доступностью фиксированных групп мембраны и допанта для взаимодействия с ними. Поэтому исследовали распределение чувствительности ПД-сенсоров к ионам треонина и $\mathrm{H}_{3} \mathrm{O}^{+}$в зависимости от объемной доли вводимых в мембрану допантов (рис. 1). Показано, что при увеличении объемной доли оксидов с сульфированной поверхностью в мембране чувствительность ПД-сенсоров к ионам $\mathrm{Thr}^{+}$, $\mathrm{Thr}^{ \pm}$возрастает, а к ионам $\mathrm{H}_{3} \mathrm{O}^{+}$снижается (рис. 1). Это обусловлено одновременным уменьшением объема внутрипорового пространства и увеличением количества центров связывания катионов и цвиттерионов аминокислоты в мембране. При таких условиях переход объемных ионов $\mathrm{Thr}^{+}, \mathrm{Thr}^{ \pm}$, видимо, возможен, а их взаимодействие с сульфогруппами на стенках пор мембраны и поверхности оксидов исключает часть протонов из ионного обмена. Наибольшее увеличение чувствительности ПД-сенсоров к ионам треонина и ее снижение к мешающим ионам (в 2-3 раза) достигается для мембран, содержащих 3-5 мас. \% $\mathrm{SiO}_{2}-\left(\mathrm{CH}_{2}\right)_{3}-\mathrm{SO}_{3} \mathrm{H}$. Это согласуется с данными, представленными в статьях $[11,12]$, согласно которым для данных образцов наблюдается снижение диффузионной проницаемости (в 2-3 раза) и увеличение проводимости по сравнению с исходным образцом, т.е. формирование вблизи поверхности допанта дебаевского слоя одноименно направленного дебаевскому слою вблизи стенок пор мембраны обеспечивает снижение объема «свободного» раствора в порах и облегчение переноса катионов.

Увеличение чувствительности ПД-сенсоров к ионам треонина и ее снижение к ионам гидроксония в результате модификации мембран обусловливает снижение пределов обнаружения треонина в 1.7-3 раза по сравнению с исходным значением (табл. 2). Использование гибридных мембран, также как и исходных, обеспечивает 
высокую стабильность отклика ПД-сенсоров в исследуемых растворах: время установления отклика - менее 1 мин, дрейф и дисперсия отклика - 7-13 мВ/ч и 18-33 мВ (табл. 2).

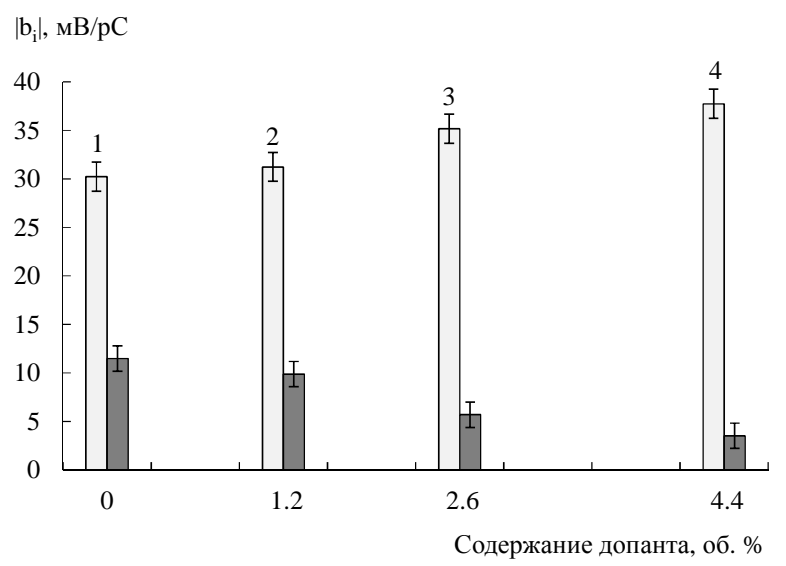

Рис. 1. Коэффициенты чувствительности ПД-сенсоров к ионам $\mathrm{Thr}^{+}, \mathrm{Thr}^{ \pm}$ ( $b_{1}$, столбцы слева) и $\mathrm{H}_{3} \mathrm{O}^{+}$( $\mathrm{b}_{2}$, столбцы справа) в зависимости от объемной доли допанта. Состав мембран МФ-4СК: 1 - исходный образец; 2 - 3 мас.\% $\mathrm{ZrO}_{2}-\mathrm{SO}_{3} \mathrm{H}$;

$$
3 \text { - } 3 \text { мас.\% } \mathrm{SiO}_{2}-\left(\mathrm{CH}_{2}\right)_{3}-\mathrm{SO}_{3} \mathrm{H} ; 4 \text { - } 5 \text { мас.\% } \mathrm{SiO}_{2}-\left(\mathrm{CH}_{2}\right)_{3}-\mathrm{SO}_{3} \mathrm{H}
$$

Таблица 2. Время установления, дрейф и дисперсия отклика ПД-сенсоров в растворе $1.0 \cdot 10^{-3} \mathrm{M} \mathrm{Thr}+1.0 \cdot 10^{-3} \mathrm{M} \mathrm{HCl}$, пределы обнаружения ионов треонина в водных растворах при $\mathrm{pH}<7$

\begin{tabular}{|c|c|c|c|c|c|}
\hline Состав мембран МФ-4СК & $\begin{array}{c}\text { Время, } \\
\text { мин }\end{array}$ & $\begin{array}{c}\text { Дрейф, } \\
\mathrm{MB} / \mathrm{ч}\end{array}$ & $\begin{array}{c}\mathrm{s}^{2}, \\
\mathrm{MB}^{2}\end{array}$ & $\begin{array}{c}\left(\Delta \varphi_{\mathrm{D}}\right)_{\text {фон }}, \\
\mathrm{MB}\end{array}$ & $\begin{array}{c}\mathrm{c}_{\min }, \\
\mathrm{M}\end{array}$ \\
\hline исходный образец & $<1$ & 7 & 26 & $208 \pm 9$ & $3.1 \cdot 10^{-7}$ \\
\hline 3 мас. \% $\mathrm{ZrO}_{2}-\mathrm{SO}_{3} \mathrm{H}$ & $<1$ & 13 & 26 & $209 \pm 10$ & $1.1 \cdot 10^{-7}$ \\
\hline 3 мас.\% $\mathrm{SiO}_{2}-\left(\mathrm{CH}_{2}\right)_{3}-\mathrm{SO}_{3} \mathrm{H}$ & $<1$ & 8 & 33 & $211 \pm 10$ & $1.8 \cdot 10^{-7}$ \\
\hline 5 мас. \% $\mathrm{SiO}_{2}-\left(\mathrm{CH}_{2}\right)_{3}-\mathrm{SO}_{3} \mathrm{H}$ & $<1$ & 12 & 18 & $210 \pm 11$ & $1.1 \cdot 10^{-7}$ \\
\hline
\end{tabular}

Учитывая результаты исследования перекрестной чувствительности и стабильности отклика ПД-сенсоров, для определения катионов и цветтерионов треонина выбраны мембраны МФ-4СК, содержащие 3-5 мас. \% $\mathrm{SiO}_{2}-\left(\mathrm{CH}_{2}\right)_{3}-\mathrm{SO}_{3} \mathrm{H}$. Cравнение результатов определения ионов $\mathrm{Thr}^{+}, \mathrm{Thr}^{ \pm}$в диапазоне концентраций от $1.0 \cdot 10^{-4}$ до $1.0 \cdot 10^{-1} \mathrm{M}$ при $\mathrm{pH}$ 1.72-4.03 с помощью исходной и выбранных гибридных мембран представлены в табл. 3. Модификация мембран МФ-4СК обеспечила снижение относительной погрешности определения ионов $\mathrm{Thr}^{+}, \mathrm{Thr}^{ \pm}$в 2.5-4 раза, при этом относительное стандартное отклонение результатов определения с их помощью - соизмеримо или несколько ниже исходного.

Таблица 3. Результаты определения ионов треонина в водных растворах при рН 1.724.03 с помощью ПД-сенсоров на основе мембран МФ-4СК: а - исходный образец; б - 3 мас.\% $\mathrm{SiO}_{2}-\left(\mathrm{CH}_{2}\right)_{3}-\mathrm{SO}_{3} \mathrm{H}$; в - 5 мас.\% $\mathrm{SiO}_{2}-\left(\mathrm{CH}_{2}\right)_{3}-\mathrm{SO}_{3} \mathrm{H}(\mathrm{n}=7-10, \mathrm{p}=0.95)$

\begin{tabular}{|c|c|c|c|c|c|c|c|c|c|}
\hline \multirow{2}{*}{$\mathrm{c}_{\mathrm{Bв}}, \mathrm{M}$} & \multicolumn{3}{|c|}{$\mathrm{c}_{\text {эксп }}, \mathrm{M}$} & \multicolumn{3}{|c|}{$\delta, \%$} & \multicolumn{3}{|c|}{$\mathrm{s}_{\mathrm{r}} \%$} \\
\cline { 2 - 11 } & $\mathrm{a}$ & $\mathrm{b}$ & $\mathrm{B}$ & $\mathrm{a}$ & 6 & $\mathrm{~B}$ & $\mathrm{a}$ & 6 & $\mathrm{~B}$ \\
\hline $1.0 \cdot 10^{-4}$ & $(0.96 \pm 0.07) \cdot 10^{-4}$ & $(1.01 \pm 0.09) \cdot 10^{-4}$ & $(1.06 \pm 0.08) \cdot 10^{-4}$ & 4 & 0.6 & 6 & 13 & 17 & 14 \\
\hline $5.0 \cdot 10^{-4}$ & $(5.1 \pm 0.3) \cdot 10^{-4}$ & $(5.2 \pm 0.3) \cdot 10^{-4}$ & $(5.1 \pm 0.3) \cdot 10^{-4}$ & 1.1 & 4 & 2 & 11 & 9 & 11 \\
\hline $1.0 \cdot 10^{-3}$ & $(1.01 \pm 0.09) \cdot 10^{-3}$ & $(1.04 \pm 0.08) \cdot 10^{-3}$ & $(1.03 \pm 0.05) \cdot 10^{-3}$ & 7 & 4 & 3 & 15 & 13 & 9 \\
\hline $5.0 \cdot 10^{-3}$ & $(5.4 \pm 0.4) \cdot 10^{-3}$ & $(5.0 \pm 0.4) \cdot 10^{-3}$ & $(5.0 \pm 0.3) \cdot 10^{-3}$ & 9 & 0.3 & 0.7 & 14 & 16 & 11 \\
\hline $1.0 \cdot 10^{-2}$ & $(0.98 \pm 0.09) \cdot 10^{-2}$ & $(0.99 \pm 0.07) \cdot 10^{-2}$ & $(0.94 \pm 0.05) \cdot 10^{-2}$ & 2 & 0.7 & 6 & 17 & 13 & 10 \\
\hline $5.0 \cdot 10^{-2}$ & $(5.3 \pm 0.3) \cdot 10^{-2}$ & $(5.1 \pm 0.3) \cdot 10^{-2}$ & $(5.3 \pm 0.3) \cdot 10^{-2}$ & 6 & 3 & 6 & 11 & 11 & 11 \\
\hline $1.0 \cdot 10^{-1}$ & $(0.85 \pm 0.08) \cdot 10^{-1}$ & $(0.96 \pm 0.09) \cdot 10^{-1}$ & $(0.94 \pm 0.06) \cdot 10^{-1}$ & 15 & 4 & 6 & 17 & 17 & 13 \\
\hline
\end{tabular}




\section{Заключение}

Разработаны перекрестно чувствительные ПД-сенсоры на основе перфторированных сульфокатионообменных мембран МФ-4СК с оксидами, поверхностно модифицированными сульфосодержащими фрагментами, в кислых растворах треонина. Показано, что чувствительность ПД-сенсоров к катионам и цвиттерионам треонина возрастает, а к ионам гидроксония снижается при увеличении объемной доли допантов в мембранах за счет одновременного уменьшения объема внутрипорового пространства и увеличения количества центров связывания ионов аналита. При таких условиях взаимодействие ионов треонина с сульфогруппами мембраны и допантов исключает часть протонов из ионного обмена. Наибольшая чувствительность ПД-сенсоров к ионам аналита и наименьшая к мешающим ионам достигается при использовании образцов, содержащих 3-5 мас. \% диоксида кремния, модифицированого 3-пропилсульфокислотными группами. Использование данных мембран обеспечило снижение относительной погрешности определения катионов и цвиттерионов треонина в диапазоне концентраций от $1.0 \cdot 10^{-4}$ до $1.0 \cdot 10^{-1} \mathrm{M}(\mathrm{pH} 1.73-4.25)$ в $2.5-4$ раза по сравнению с исходным образцом.

Авторы выражают благодарность д.х.н., проф., член.-корр. РАН Ярославщеву А.Б. и к.Х.н. Сафроновой Е.Ю. за предоставление образцов мембран и помощь в обсуждении полученных результатов. Исследование выполнено за счет гранта Российского научного фонда (проект № 15-13-10036).

\section{Список литературы}

1. Tsukahara Sh., Nanzai B., Igawa M. // Journal of Membrane Science. 2013. Vol. 448. pp. 300-307.

2. Васильева В.И., Голева Е. А., Селеменев В.Ф. // Журнал физической химии. 2016. Т. 90. № 10. C. 1548-1557.

3. Kattan Readi O.M., Kuenen H.J., Zwijnenberg H.J., Nijmeijer K. // Journal of Membrane Science. 2013. Vol. 443. pp. 219-226.

4. Елисеева Т.В., Харина А.Ю. // Электрохимия. 2015. Т. 51. № 1. С. 74-80.

5. Khaleghi F., Irai A.E., Gupta V.K., Agarwal Sh. et al. // Journal of Molecular Liquids. 2016. Vol. 223. pp. 431-435.

6. Yokus Ö. A., Kardas F., Akyıldırım O., Eren T. et al. // Sensors and Actuators B: Chemical. 2016. Vol. 233. pp. 47-54.

7. Зяблов А.Н., Ковалева Н.В., Жиброва Ю.А., Красникова О.П. и др. // Журнал аналитической химии. 2013. Т. 68. № 4. С. 331333.

\section{References}

1. Tsukahara Sh., Nanzai B., Igawa M., pp. 300-307. DOI:10.1016/j.memsci.2013. Journal of Membrane Science, 2013, Vol. 448, 06.062.
8. Lata S., Pundir C.S. // International Journal of Biological Macromolecules. 2013. Vol. 54. pp. 250-257.

9. Qiao Xia, Yihan Huang, Xia Lin, Shu Zhu et al. // Biochemical Engineering Journal. 2016. Vol. 113. pp. 1-6.

10.Паршина А.В., Денисова Т.С., Бобрешова О.В. // Мембраны и мембранные технологии. 2016. Т. 6. № 4. С. 329-350.

11.Сафронова Е.Ю., Паршина А.В., Янкина К.Ю., Рыжкова Е.А. и др. // Мембраны и мембранные технологии. 2017. Т. 7. № 2. С. 110-116.

12.Prikhno I.A., Safronova E.Yu., Yaroslavtsev A.B. // International Journal of Hydrogen Energy. 2016. Vol. 41. pp. 15585-15592.

13. Mahreni A., Mohamad A.B., Kadhum A.A.H., Daud W.R.W. et al. // Journal of Membrane Science. 2009. Vol. 327. pp. 32-40. 
2. Vasil'eva V.I., Goleva E.A., Selemenev V.F., Russian Journal of Physical Chemistry A. 2016, Vol. 90, No 10, pp. 2035-2043. DOI: $10.1134 / \mathrm{S} 0036024416100277$.

3. Kattan Readi O.M., Kuenen H.J., Zwijnenberg H.J., Nijmeijer K., Journal of Membrane Science, 2013, Vol. 443, pp. 219-226. DOI: 10.1016/j.memsci.2013.04.045.

4. Eliseeva T.V., Kharina A.Y., Russian Journal of Electrochemistry, 2015, Vol. 51. No 1. pp. 63-69. DOI: 10.1134/S1023193515010048.

5. Khaleghi F., Irai A.E., Gupta V.K., Agarwal Sh. et al., Journal of Molecular Liquids. 2016. Vol. 223. pp. 431-435. DOI: 10.1016/j.molliq.2016.08.058.

6. Yokus Ö.A., Kardas F., Akyıldırım O., Eren T. et al., Sensors and Actuators B: Chemical, 2016, Vol. 233, pp. 47-54. DOI:10.1016/j.snb.2016.04.050.

7. Zyablov A.N., Kovaleva N.V., Selemenev V.F., Zhibrova Y.A. et al., Journal of Analytical Chemistry, 2013, Vol. 68. No 4. pp. 305306. DOI: $10.1134 / \mathrm{S} 1061934813040138$.

Титова Татьяна Сергеевна - аспирант, кафедра аналитической химии, Воронежский государственный университет, Воронеж

Рыжих Елена Ивановна - студент, кафедра аналитической химии, Воронежский государственный университет, Воронеж

Паршина Анна Валерьевна - д.х.н., доцент, кафедра аналитической химии, Воронежский государственный университет, Воронеж, parshina_ann@mail.ru

Бобрешова Ольга Владимировна - д.х.н., профессор, главный научный сотрудник, кафедра аналитической химии, Воронежский государственный университет, Воронеж
8. Lata S., Pundir C.S., International Journal of Biological Macromolecules, 2013, Vol. 54, pp. 250-257.

DOI:10.1016/j.ijbiomac.2012.12.004.

9. Qiao Xia, Yihan Huang, Xia Lin, Shu Zhu et al., Biochemical Engineering Journal, 2016, Vol. 113, pp. 1-6. DOI: 10.1016/j.bej.2016.05.003.

10.Parshina A.V., Denisova T.S., Bobreshova O.V., Petroleum Chemistry, 2016, Vol. 56, No 11, pp. 987-1005. DOI: 10.1134/S096554411611013X.

11.Safronova E.Yu., Parshina A.V., Yankina K.Yu., Ryzhkova E.A. et al., Petroleum Chemistry, 2017, Vol. 57, No 4. pp. 327-333. DOI: 10.1134/S0965544117040077.

12.Prikhno I.A., Safronova E.Yu., Yaroslavtsev A.B., International Journal of Hydrogen Energy, 2016, Vol. 41, pp. 15585-15592. DOI.10.1016/j.ijhydene.2016.04.100.

13.Mahreni A., Mohamad A.B., Kadhum A.A.H., Daud W.R.W. et al., Journal of Membrane Science, 2009,Vol. 327,pp. 32-40. DOI:10.1016/j.memsci.2008.10.048.

Titova Tatyana S. - postgraduate student, department of analytical chemistry, Voronezh State University, Voronezh

Ryzhih Elena I. - student, department of analytical chemistry, Voronezh State University, Voronezh

Parshina Anna V. - Dr.Sci. (Chemistry), docent, department of analytical chemistry, Voronezh State University, Voronezh, parshina_ann@mail.ru

Bobreshova Olga V. - Dr.Sci. (Chemistry), professor, chief scientific researcher, department of analytical chemistry, Voronezh State University, Voronezh 